\title{
A ROSAT PSPC catalogue of X-ray sources in the SMC region ${ }^{\star}$
}

\author{
F. Haberl ${ }^{1}$, M.D. Filipović ${ }^{1,2,3}$, W. Pietsch ${ }^{1}$, and P. Kahabka ${ }^{4,5}$ \\ 1 Max-Planck-Institut für extraterrestrische Physik, Giessenbachstrasse, 85748 Garching, Germany \\ 2 University of Western Sydney Nepean, P.O. Box 10, Kingswood, NSW 2747, Australia \\ 3 Australia Telescope National Facility, CSIRO, P.O. Box 76, Epping, NSW 2121, Australia \\ 4 Astronomical Institute, University of Amsterdam, Kruislaan 403, NL-1098 SJ Amsterdam, The Netherlands \\ 5 Center for High Energy Astrophysics, University of Amsterdam, Kruislaan 403, NL-1098 SJ Amsterdam, The Netherlands
}

Received October 8; accepted November 19, 1999

\begin{abstract}
We present a catalogue of 517 discrete X-ray sources in a $6^{\circ} \times 6^{\circ}$ field covering the Small Magellanic Cloud (SMC). The catalogue was derived from the pointed ROSAT PSPC observations performed between October 1991 and May 1994 and is complementary to the Large Magellanic Cloud (LMC) catalogue published by Haberl \& Pietsch (1999). We followed the same identification scheme and used, among other information, X-ray hardness ratios and spatial extent to classify unknown sources as candidates for active galactic nuclei (AGN), foreground stars, supernova remnants (SNRs), supersoft sources (SSSs) and $\mathrm{X}$-ray binaries. For 158 sources a likely source type is given, from which 46 sources are suggested as background AGN (including candidates resulting from a comparison of X-ray and radio images). Nearly all of the X-ray binaries known in the SMC were detected in ROSAT PSPC observations; most of them with luminosities below $10^{36} \mathrm{erg} \mathrm{s}^{-1}$ suggesting that the fraction of high luminosity X-ray binary systems in the Magellanic Clouds (MCs) is not significantly larger than in our galaxy. Seventeen X-ray sources are associated with SNRs found in earlier work and we suggest here two additional extended sources as SNR candidates. Three very soft sources are newly classified as SSSs from which one is identified with the symbiotic star LIN 358 in the SMC.
\end{abstract}

Key words: catalogues — ISM: supernova remnants galaxies: magellanic clouds — galaxies: stellar content X-rays: galaxies - X-rays: stars

\section{Send offprint requests to: F. Haberl}

e-mail: fwh@mpe.mpg.de

* Table 2 is only available in electronic form at CDS via anonymous ftp to cdsarc.u-strasbg.fr (130.79.128.5) or via http://cdsweb.u-strasbg.fr/Abstract.html

\section{Introduction}

The most comprehensive X-ray studies of the MCs before the launch of ROSAT were performed using the imaging instruments on the Einstein satellite. After a final unified analysis of the IPC data, Wang et al. (1991) and Wang \& Wu (1992, hereafter WW92) published catalogues of 105 and 70 discrete X-ray sources in the LMC and SMC regions, respectively. With ROSAT (Trümper 1982) the amount of high angular resolution X-ray data from the MCs increased considerably. Many pointed observations from various scientific programs, in particular using the PSPC imaging detector $(0.1-2.4 \mathrm{keV}$; Pfeffermann et al. 1986), are available to completly survey both Clouds. Haberl \& Pietsch (1999, hereafter HP99) analyzed more than 200 PSPC pointed observations covering 59 square degrees in the field towards the LMC. They compiled a catalogue of 758 point and point-like X-ray sources and used the properties of identified sources to classify nearly 100 sources as new candidates for SNRs, X-ray binaries, SSSs, foreground stars and background AGN.

Kahabka \& Pietsch (1996) conducted a PSPC survey of the SMC covering 9 square degrees and Kahabka et al. (1999, hereafter KPFH99) published a first ROSAT PSPC catalogue with $248 \mathrm{X}$-ray sources. The number of PSPC observations finally available in the ROSAT archive is twice of that used by KPFH99. We therefore analyzed the whole set of PSPC pointings towards the SMC to derive a complete catalogue of PSPC sources in the SMC region as was provided by HP99 for the LMC. To obtain this new complementary catalogue the analysis was done the same way as in HP99 (Sect. 2). In Sect. 3 we present the ROSAT PSPC catalogue of SMC sources together with a classification based on various properties. 


\section{ROSAT PSPC observations and analysis}

ROSAT performed 31 pointed PSPC observations with at least $200 \mathrm{~s}$ of exposure in a $6^{\circ} \times 6^{\circ}$ area centered on the position $\mathrm{RA}=01^{\mathrm{h}} 00^{\mathrm{m}} 00^{\mathrm{s}}$ Dec $=-73^{\circ} 30^{\prime} 00^{\prime \prime}$ in the SMC between 1990 and 1994. Most of the observations (summarized in Table 1) had exposures between $5 \mathrm{ks}$ and $20 \mathrm{ks}$ and in one case reached $64.6 \mathrm{ks}$ (centered on $\mathrm{CF}$ Tuc).

An X-ray image of the SMC produced from merged ROSAT PSPC data in the energy band $0.5-2.0 \mathrm{keV}$ is shown in Fig. 1. The image is exposure corrected but not background subtracted and covers an exposed area of $\sim 18$ square degrees. Time intervals with a total count rate of more than $15 \mathrm{cts} \mathrm{s}^{-1}$ were excluded to keep the background at the lowest possible level and uniform in the image. The image was binned to $1536 \times 1536$ pixels of $15^{\prime \prime} \times 15^{\prime \prime}$ size and smoothed with an intensity dependent Gaussian filter before the exposure correction (stronger smoothing at the low intensity levels and no smoothing above 15 counts per pixel to avoid smearing out the stronger point sources). The still visible blurring is caused by the telescope when sources are observed far off-axis (see the sources at the rim of the exposures). For this reason conclusions on possible diffuse emission in the SMC require a more careful quantitative study beyond the scope of this paper. A wealth of X-ray sources is detected in the ROSAT PSPC data with most sources concentrated in the northern part of the SMC. The combination of three images in the energy bands $0.1-0.4 \mathrm{keV}, 0.5-0.9 \mathrm{keV}$ and $0.9-2.0 \mathrm{keV}$ into a red-green-blue (RGB) image is shown in Fig. 2. The colours illustrate the different X-ray spectra of various source types. Sources with soft X-ray spectra (high intensities in the "red" $0.1-0.4 \mathrm{keV}$ band) like foreground stars and in particular SSSs appear in orange/red. SNRs are characterized by harder spectra and green to blue colours. Absorbed AGN and X-ray binaries show the hardest $\mathrm{X}$-ray spectra and blue colours in the RGB image.

We used the EXSAS software package (Zimmermann et al. 1994) for the analysis of the individual pointings and followed the same lines as in HP99 for the LMC. However, the 31 SMC observations covered 16 different fields and pointings in the same direction were merged to gain sensitivity. In these cases both merged and individual images were run through the source detection procedure. After the final visual inspection of the source list a catalogue of 517 point-like and weakly extended sources was obtained with source properties as given in the LMC catalogue of HP99. Both catalogues comprise complementary lists of sources detected with the ROSAT PSPC in the fields of the MCs.
Table 1. ROSAT PSPC observations of the SMC region

\begin{tabular}{|c|c|c|c|c|}
\hline ID & $\begin{array}{c}\text { Exp. } \\
\text { s }\end{array}$ & $\begin{array}{l}\text { Date } \\
\text { begin end }\end{array}$ & \multicolumn{2}{|c|}{$(\mathrm{J} 2000.0)$} \\
\hline $201094 p$ & 64636 & 921002921111 & 005048.00 & -744648.0 \\
\hline $300369 p$ & 5640 & 930928931101 & 012255.02 & $\begin{array}{lll}-75 & 21 & 00.0\end{array}$ \\
\hline $300369 \mathrm{p}-1$ & 213 & 940401940401 & 012255.02 & $\begin{array}{lll}-75 & 21 & 00.0\end{array}$ \\
\hline $400022 p$ & 16634 & 911007911008 & $01 \quad 1704.08$ & $\begin{array}{lll}-73 & 26 & 24.0\end{array}$ \\
\hline $400022 \mathrm{p}-1$ & 8985 & 920930921002 & $01 \quad 1704.08$ & -732624.0 \\
\hline $400022 \mathrm{p}-2$ & 11864 & 930603930604 & $01 \quad 1704.08$ & -732624.0 \\
\hline $400149 p$ & 6281 & 920428920603 & 003719.02 & $\begin{array}{lll}-72 & 14 & 24.0\end{array}$ \\
\hline $400299 p$ & 5121 & 930410930424 & $0037 \quad 19.02$ & $-72 \quad 1424.0$ \\
\hline $400299 \mathrm{p}-1$ & 1677 & 931002931009 & $0037 \quad 19.02$ & $\begin{array}{llll}-72 & 14 & 24.0\end{array}$ \\
\hline $400299 \mathrm{p}-2$ & 2311 & 940503940505 & $0037 \quad 19.02$ & $\begin{array}{llll}-72 & 14 & 24.0\end{array}$ \\
\hline $400300 p$ & 5214 & 930329930330 & 005833.06 & $\begin{array}{llll}-71 & 36 & 00.0\end{array}$ \\
\hline $400300 \mathrm{p}-1$ & 7199 & 931001931009 & 005833.06 & $\begin{array}{llll}-71 & 36 & 00.0\end{array}$ \\
\hline $400300 \mathrm{p}-2$ & 4111 & 940505940505 & 005833.06 & $\begin{array}{lll}-71 & 36 & 00.0\end{array}$ \\
\hline $500142 \mathrm{p}$ & 4909 & 930512930513 & 010402.04 & $\begin{array}{lll}-72 & 01 & 48.0\end{array}$ \\
\hline $500249 p$ & 19262 & 931105931109 & 004640.08 & $-73 \quad 1236.0$ \\
\hline $500250 p$ & 20860 & 931014931029 & 010438.04 & -720523.5 \\
\hline $500251 p$ & 2093 & 931130931130 & 005119.02 & -732400.0 \\
\hline $600195 \mathrm{p}-0$ & 16644 & 911008911103 & $0058 \quad 12.00$ & $\begin{array}{lll}-72 & 16 & 48.0\end{array}$ \\
\hline $600195 \mathrm{p}-1$ & 9443 & 920417920427 & 005812.00 & $\begin{array}{lll}-72 & 16 & 48.0\end{array}$ \\
\hline $600196 \mathrm{p}-0$ & 1303 & 911009911102 & 005045.06 & $\begin{array}{lll}-73 & 13 & 48.0\end{array}$ \\
\hline $600196 \mathrm{p}-1$ & 22223 & 920415920425 & 005045.06 & $\begin{array}{lll}-73 & 13 & 48.0\end{array}$ \\
\hline $600197 p$ & 21494 & 911016911019 & 011324.00 & $\begin{array}{lll}-72 & 49 & 12.0\end{array}$ \\
\hline $600452 p$ & 14207 & 930410930425 & 010555.02 & -723335.5 \\
\hline $600452 \mathrm{p}-1$ & 16663 & 931001931014 & 010555.02 & -723335.5 \\
\hline $600453 p$ & 17593 & 930509930512 & 005428.08 & -724536.0 \\
\hline $600454 p$ & 9727 & 921205921208 & 004255.02 & -733824.0 \\
\hline $600454 \mathrm{p}-1$ & 8299 & 930405930426 & 004255.02 & -733824.0 \\
\hline $600455 p$ & 3561 & 921206921206 & 010116.08 & $\begin{array}{lll}-71 & 49 & 12.0\end{array}$ \\
\hline $600455 \mathrm{p}-1$ & 1721 & 930416930422 & 010116.08 & $\begin{array}{lll}-71 & 49 & 12.0\end{array}$ \\
\hline $600455 \mathrm{p}-2$ & 4595 & 931007931010 & 010116.08 & $\begin{array}{lll}-71 & 49 & 12.0\end{array}$ \\
\hline $600455 \mathrm{p}-3$ & 4129 & 940504940505 & $\begin{array}{lll}01 & 01 & 16.08\end{array}$ & -714912.0 \\
\hline
\end{tabular}

\section{The catalogue of discrete X-ray sources}

Table 2 with the full SMC catalogue is available electronically and contains the following information for each X-ray source: (1) source number, (2) likelihood of existence (maximum value from the five detection energy bands) $\mathrm{ML}_{\text {exi }}$, (3) vignetting corrected net exposure, (4) and (5) source coordinates RA and Dec (J2000.0) derived from observation and energy band with smallest position error, (6) statistical $90 \%$ confidence error on the X-ray position with an additioanl systematic uncertainty of about $7^{\prime \prime},(7)$ PSPC $0.1-2.4 \mathrm{keV}$ count rate and error, (8) and (9) hardness ratios (defined as $\mathrm{HR} 1=(\mathrm{H}-\mathrm{S}) /(\mathrm{S}+\mathrm{H})$ and $\mathrm{HR} 2=$ $(\mathrm{H} 2-\mathrm{H} 1) /(\mathrm{H} 1+\mathrm{H} 2)$ where $\mathrm{S}, \mathrm{H}, \mathrm{H} 1$ and $\mathrm{H} 2$ denote count rates in the $0.1-0.4 \mathrm{keV}, 0.5-2.0 \mathrm{keV}, 0.5-0.9 \mathrm{keV}$ and $0.9-2.0 \mathrm{keV}$ bands, respectively; not calculated in cases where not all required count rates are available), (10) source extent from same detection as source position, (11) likelihood for the extent, (12) ROSAT observation identifier (a "+" at the end marks merged observations), (13) off-axis angle, (14) distance to detector window support structure or detector rim (WSS), (15) number of nearest (within $60^{\prime \prime}$ ) source in KPFH99 (see Sect. 3.1) and (16) remark (cf. Table 1 in HP99). We note here that the count rates given in KPFH99 and here can considerably differ for sources repeatedly covered by PSPC observations. Since the main goal of this work is to identify sources, we selected the detection with smallest error on the X-ray 


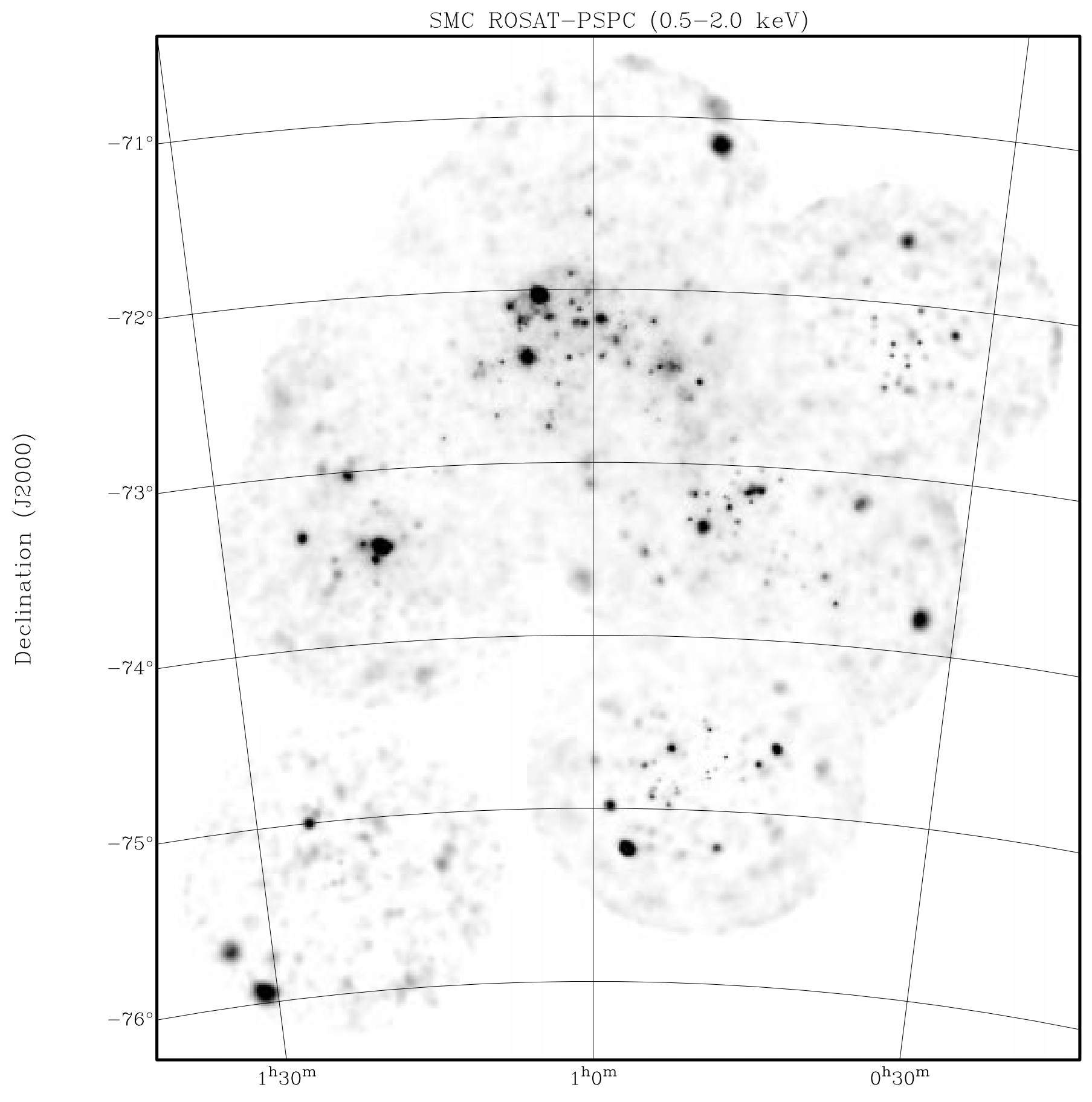

Right Ascension (J2000)

Fig. 1. Exposure corrected $0.5-2.0 \mathrm{keV}$ image of the combined ROSAT PSPC observations in the SMC region

position which may result from a different PSPC observation not used by KPFH99.

The spatial distribution of the 517 PSPC sources in the catalogue is shown in Fig. 3. Haberl \& Pietsch (1999) have used the LMC catalogue to identify known sources and classify unknown sources according to the properties of the identified samples. Again we follow the same procedures for the SMC region.

\subsection{Comparison with previous $X$-ray surveys}

In Table 3 we summarize 50 PSPC sources which have an Einstein IPC counterpart (from Tables 2A and B in WW92) within $90^{\prime \prime}$. The IPC and PSPC count rates are compared in Fig. 4 where the sources identified with a SNR are marked with a circle. A linear fit to the SNR count rates gives a mean conversion factor of 2.8 for IPC 


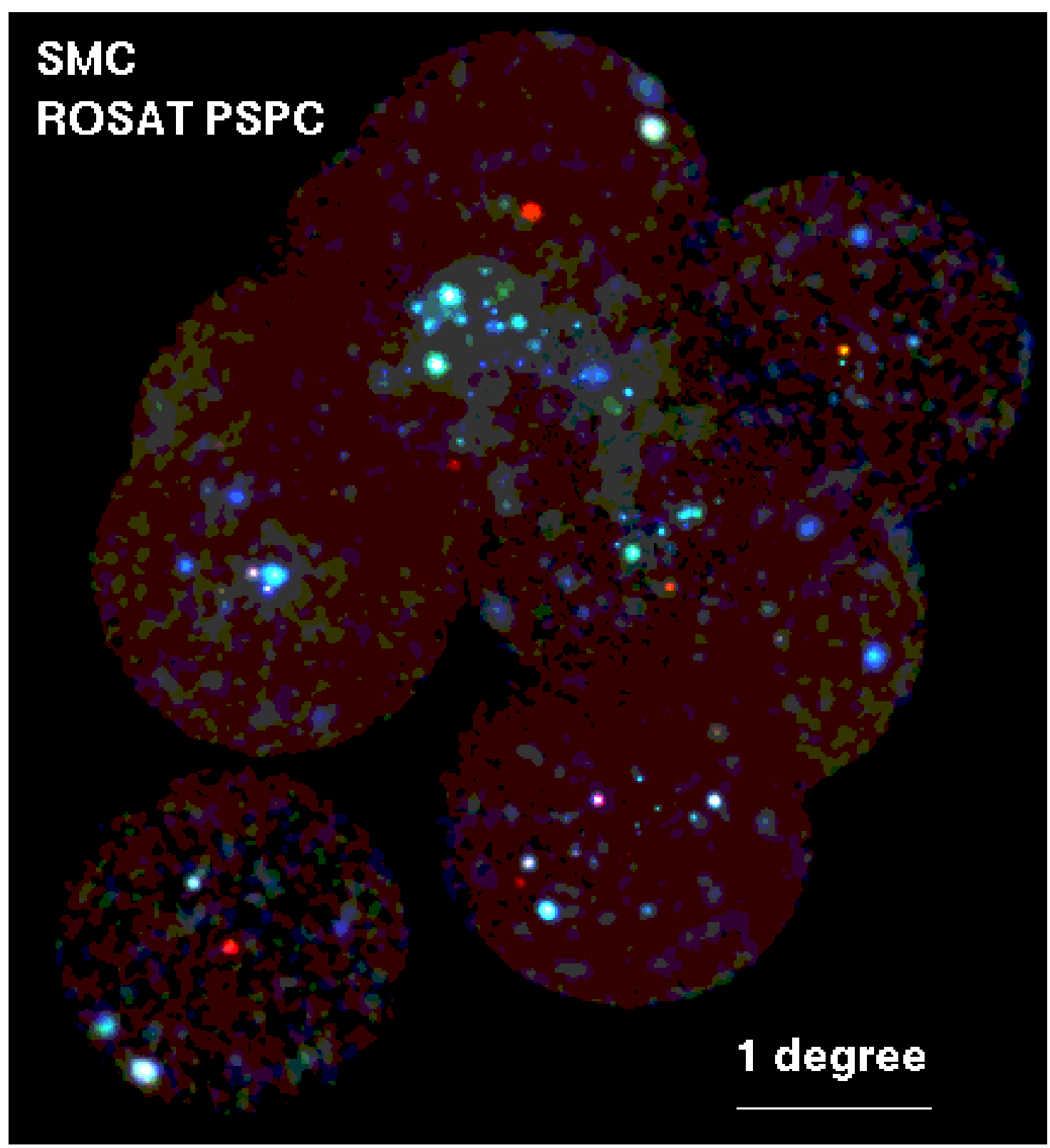

Fig. 2. PSPC images in the energy bands $0.1-0.4 \mathrm{keV}, 0.5-0.9 \mathrm{keV}$ and $0.9-2.0 \mathrm{keV}$ combined into a red-green-blue (RGB) colour coded image

to PSPC count rates, somewhat smaller than found in the LMC which is compatible with a slightly higher foreground absorption. The high mass X-ray binary (HMXB) pulsar SMCX-1 and the RSCVn type foreground star HD 5303 were significantly brighter during the ROSAT observations (because the best source detection with smallest position error enters the catalogue, detections during bright source state are preferentially selected) while the pulsar RX J0051.8-7231 probably associated with the IPC source 0050.1-7248 was bright during the Einstein observations. The unidentified ROSAT source 320 may be associated with 0101.3-7300 (a relatively large correlation distance leaves some doubt) in which case the classifica- tion as foreground star suggests a flare during the IPC observation.

A correlation of the PSPC catalogue from the pointed observations with the Bright Source Catalogue (BSC) of the ROSAT all-sky survey (Voges et al. 1996, 1999) yields 16 sources within a distance of $60^{\prime \prime}$ which are summarized in Table 4. Strong variability between survey and pointed observations was found for the two brightest sources of this sample (SMCX-1 and HD 5303) and for the SSS RXJ 0048.4-7332 which was more than a factor 20 fainter during the pointing with identifier 500251p than during survey. However, during other pointings RXJ 0048.4-7332 
Table 2. Excerpt of the catalogue of 517 ROSAT PSPC sources from a $6^{\circ} \times 6^{\circ}$ field centered on the SMC

\begin{tabular}{|c|c|c|c|c|c|c|c|c|c|c|}
\hline 1 & 2 & 3 & 4 & 5 & 6 & 7 & 8 & 9 & 10 & 11 \\
\hline No & $\mathrm{ML}_{\text {exi }}$ & $\begin{array}{l}\text { Exp. } \\
{[\mathrm{s}]}\end{array}$ & ${ }^{\mathrm{RA}}$ & $\begin{array}{l}\text { Dec } \\
00.0)^{-}\end{array}$ & $\begin{array}{r}r_{90} \\
{\left[{ }^{\prime \prime}\right]}\end{array}$ & $\begin{array}{r}\text { Count Rate } \\
{\left[\operatorname{cts~s}^{-1}\right]}\end{array}$ & HR1 & HR2 & $\begin{array}{r}\text { Extent } \\
{\left[{ }^{\prime \prime}\right]}\end{array}$ & $\mathrm{ML}_{\text {ext }}$ \\
\hline 49 & 12.2 & 15341 & 010249.5 & -713709 & 42.6 & $3.41 \mathrm{e}-03 \pm 4.4 \mathrm{e}-03$ & $1.00 \pm 0.84$ & $-1.00 \pm 1.69$ & 0.0 & 0.0 \\
\hline 50 & 47.1 & 14862 & 005547.3 & -713803 & 10.0 & $4.90 \mathrm{e}-03 \pm 7.4 \mathrm{e}-04$ & $-0.22 \pm 0.15$ & $-0.21 \pm 0.22$ & 0.0 & 0.0 \\
\hline 51 & 25.7 & 15176 & 005941.7 & -713815 & 14.5 & $2.94 \mathrm{e}-03 \pm 6.1 \mathrm{e}-04$ & $-1.00 \pm 0.12$ & & 0.0 & 0.0 \\
\hline 52 & 326.6 & 9397 & 003656.9 & -713815 & 17.5 & $3.86 \mathrm{e}-02 \pm 2.8 \mathrm{e}-03$ & $1.00 \pm 0.25$ & $0.38 \pm 0.06$ & 3.4 & 19.1 \\
\hline 53 & 21360.4 & 3470 & 005911.3 & -713845 & 2.8 & $8.38 \pm 5.0 \mathrm{e}-02$ & $0.48 \pm 0.01$ & $0.17 \pm 0.01$ & 1.1 & 14.5 \\
\hline
\end{tabular}

\begin{tabular}{lrrrl}
\hline \multicolumn{1}{c}{12} & 13 & 14 & 15 & \multicolumn{1}{c}{16} \\
\hline ROR & $\delta$ & $d_{\text {rib }}$ & KPFH99 & Remark \\
& {$\left[^{\prime}\right]$} & {$[\mathrm{FWHM}]$} & & \\
\hline $500250 \mathrm{p}$ & 29 & 1.3 & 0 & AGN? 13 cm \\
$400300 \mathrm{p}+$ & 13 & 6.0 & 109 & fg Star F5/F6V, HD 5572 (SIM) \\
$400300 \mathrm{p}+$ & 6 & 20.6 & 141 & SSS? RXJ0059.6-7138, PN LIN357 (KPFH99) \\
$400299 \mathrm{p}+$ & 36 & 1.8 & 19 & AGN? [hard] [nonstar] Radio SMC B0034-7155 (FJW97) 13 cm jets \\
$500142 \mathrm{p}$ & 32 & 4.3 & 0 & HMXB Be/X RXJ0059.2-7138, soft pulsar (H94, SC96) \\
\hline
\end{tabular}

was detected with count rates similar to the survey intensity (see Kahabka et al. 1994).

The first SMC catalogue based on ROSAT PSPC data and published by KPFH99 is less complete than the catalogue presented here mainly due to the limited number of observations and the more restricted field of view ( $45^{\prime}$ vs. $\left.52^{\prime}\right)$ used for the analysis. A correlation of the two catalogues shows that 209 sources have a counterpart within $60^{\prime \prime}$. For these the source number from KPFH99 is given in Table 2. The 39 sources of KPFH99 without counterpart in the new catalogue are summarized in Table 5. KPFH99 marked 15 of these with "D" as probable artifacts close to the WSS and we confirm that they are not real. Four other sources with relatively high likelihood are probably also false detections: source 223 in KPFH99 is the most extended one and not visible on the PSPC images; sources 75, 165 and 185 are near the WSS. Two sources $(25,186)$ are also near the WSS but may be real. Another 17 sources have likelihood values smaller than 15 , close to our acceptance threshold and a slightly different background map used in the analysis is probably the reason why they are not detected in our analysis. Source 60 may be resolved into two X-ray sources (413 and 419 in the present catalogue) in an observation which was not used by KPFH99 but has source 60 closer to its field center. Source 413 coincides with a known SNR showing radio emission. Inspecting the ATCA $\lambda=13 \mathrm{~cm}$ image (see below) shows that the radio emission is associated with source 413 suggesting that 419 is a separate source.

\subsection{Source identification and classification}

Source identifications were mainly obtained by crosscorrelation of the SMC catalogue with the SIMBAD data base operated at CDS. The derived samples of known $\mathrm{X}$-ray sources show identical properties to those of the LMC and therefore we used similar classification criteria as for the LMC sources (Table 6; cf. Table 3 of HP99). As only exception, the selection for SSS was expanded to HR1+EHR $1<-0.70$ which includes all sources with $\mathrm{HR} 2+\mathrm{EHR} 2<-0.70$.

To select objects with high X-ray to optical flux ratio we enlarged the $f_{\mathrm{x}} / f_{\text {opt }}$ classification used for the LMC. By using the optically brightest object in the error circle (to be conservative the $90 \%$ confidence error including the systematic error was multiplied by 1.5) a lower limit for the X-ray to optical flux ratio is derived. For this purpose optical $R$ and $B$ magnitudes were taken from the USNOA1.0 catalogue produced by the US Naval Observatory. Finally selecting objects with $\log \left(f_{\mathrm{x}} / f_{\mathrm{B}}\right)$ greater than 0 excludes foreground stars and we classified these as [nonstar.] Sources of this type in the observed regions outside the main regions of the SMC are candidates for background sources and if south of $-74^{\circ} 30^{\prime}$ were in addition classified as $[\mathrm{AGN}]$. However, we note that the H I emission of the SMC (Stanimirovic et al. 1999) extends that far south and finding X-ray binaries there is not completely ruled out.

Figures 5, 6 and 7 illustrate the selection criteria used for source extent, hardness ratios and X-ray to optical flux ratio. As in the LMC, the identified well known SNRs show the highest extent likelihoods and the two classified SNR candidates (mainly from hardness ratio selection) are found with significant X-ray extent. Also similar to the LMC the different source types separate in different regions of the HR diagram (Fig. 6) which can be divided into the same areas as in the LMC case. There is one object (source 380) with hardness ratios compatible to that 


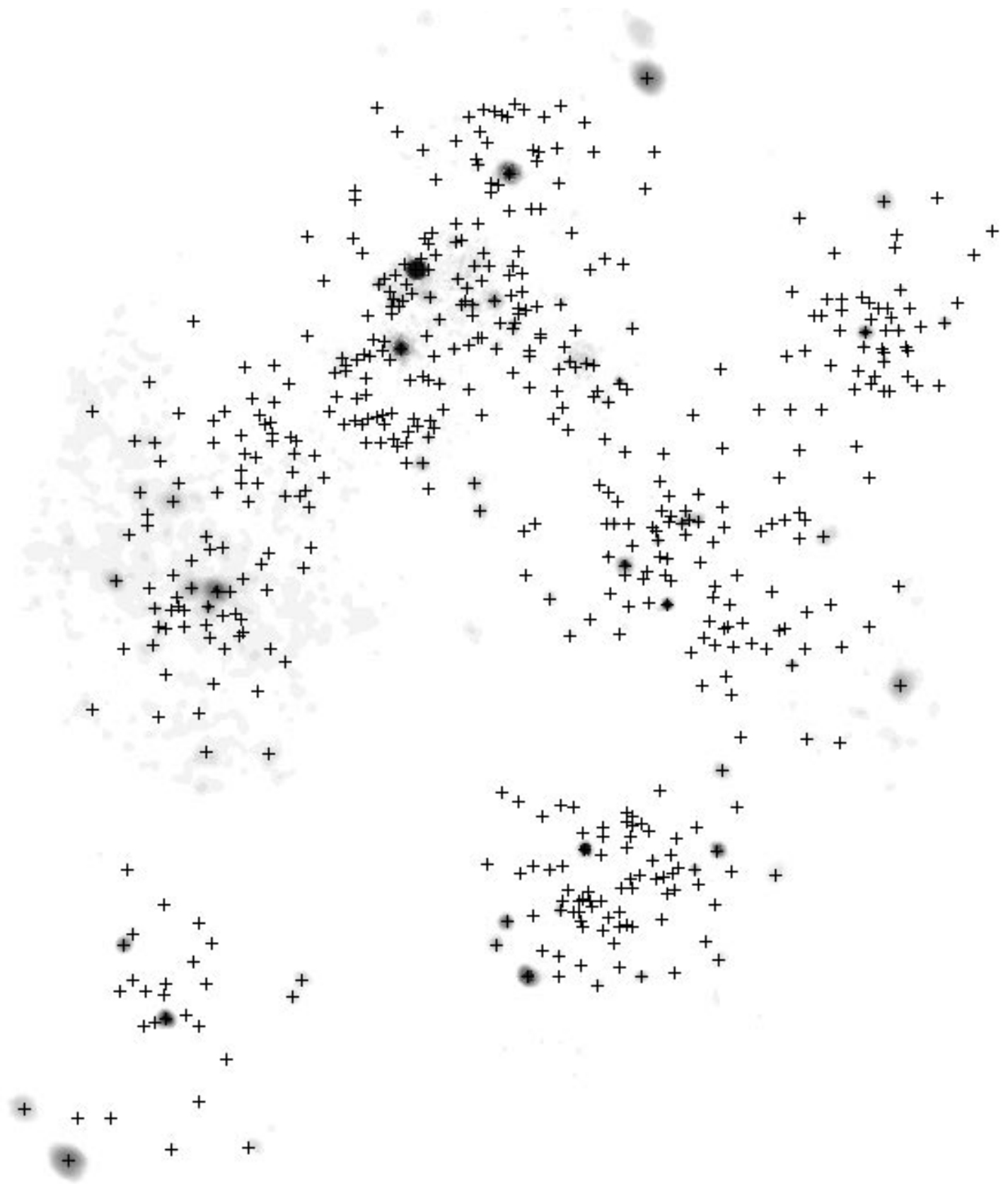

Fig. 3. Distribution of the 517 X-ray sources detected with the ROSAT PSPC. The source positions are plotted on a weak grey scale image $(0.1-2.4 \mathrm{keV})$ similar to Fig. 1 for orientation

of foreground stars which was classified as [nonstar] due to its high $\mathrm{X}$-ray to optical flux ratio. A nearby radio source suggests the source as AGN candidate. The X-ray binary with HR1 near 0.5 marks the peculiar soft X-ray pulsar (source 53, H94). Foreground stars are well recognized from their hardness ratios and also from their low X-ray to optical flux ratio, i.e. typically $\log \left(f_{\mathrm{x}} / f_{\text {opt }}\right)<-1$ (Fig. 7). Most of the objects with high $f_{\mathrm{x}} / f_{\text {opt }}$ have errors on HR1 larger than 0.25 and are not shown in (Fig. 7).
Table 7 summarizes the properties of 158 identified and classified sources sorted by source class. The classification (including candidates) is complete for the 44 brightest sources down to a PSPC count rate of $0.02 \mathrm{cts} \mathrm{s}^{-1}$. This sample consists of $27 \%$ foreground stars, $16 \%$ background objects, 20\% high mass X-ray binaries, 16\% SNRs, 9\% SSSs in the SMC and 9\% sources which are probably either AGN or HMXBs. We expect at lower intensity levels less foreground stars but more background AGN. 
Table 3. PSPC sources detected with the Einstein IPC

\begin{tabular}{|c|c|c|c|c|c|c|c|}
\hline \multirow{3}{*}{$\frac{1}{\text { No }}$} & \multicolumn{2}{|c|}{2} & \multirow{3}{*}{$\begin{array}{c}3 \\
d \\
{\left[{ }^{\prime \prime}\right]}\end{array}$} & \multirow{3}{*}{$\begin{array}{c}4 \\
r_{90} \\
{\left[{ }^{\prime \prime}\right]}\end{array}$} & \multirow{3}{*}{$\begin{array}{r}5 \\
\text { PSPC Count Rate } \\
{\left[\text { cts s }^{-1}\right]}\end{array}$} & \multirow{3}{*}{$\begin{array}{c}6 \\
\text { IPC Rate } \\
{\left[\operatorname{cts~s}^{-1}\right]}\end{array}$} & \multirow{3}{*}{$\frac{7}{\text { Remarks }}$} \\
\hline & \multicolumn{2}{|c|}{ WW92 } & & & & & \\
\hline & No & Name & & & & & \\
\hline 8 & 23 & $0049.0-7125$ & 15.5 & 20.2 & $2.04 \mathrm{e}-01 \pm 1.1 \mathrm{e}-02$ & 0.046 & fg Star F5V, HD 5028 (WW92) \\
\hline 47 & 43 & 0056.8-7152 & 2.6 & 1.3 & $3.68 \mathrm{e}-01 \pm 4.9 \mathrm{e}-03$ & 0.047 & SSS 1E0056.8-7154, PN LIN333 (KP96) \\
\hline 94 & 32 & 0051.3-7213 & 62.2 & 30.2 & $1.21 \mathrm{e}-02 \pm 2.1 \mathrm{e}-03$ & 0.006 & \\
\hline 107 & 51 & 0102.3-7218 & 13.4 & 0.7 & $2.24 \pm 2.1 \mathrm{e}-02$ & 0.807 & SNR 0102-72.3 (WW92,FPW99) $13 \mathrm{~cm}$ \\
\hline 114 & 41 & $0056.2-7219$ & 58.3 & 7.9 & $3.03 \mathrm{e}-03 \pm 5.0 \mathrm{e}-04$ & 0.002 & HMXB? AXJ0058-72.0, pulsar (YK98a) \\
\hline 121 & 46 & $0059.8-7220$ & 37.6 & 7.2 & $8.93 \mathrm{e}-03 \pm 8.5 \mathrm{e}-04$ & 0.003 & \\
\hline 125 & 54 & 0104.5-7222 & 52.1 & 3.6 & $3.36 \mathrm{e}-02 \pm 1.5 \mathrm{e}-03$ & 0.008 & SNR 0104-72.3 (WW92) $13 \mathrm{~cm}$ \\
\hline 143 & 50 & $0101.5-7225$ & 17.5 & 3.4 & $1.54 \mathrm{e}-02 \pm 9.6 \mathrm{e}-04$ & 0.013 & HMXB Be/X SAXJ0103.2-7209, pulsar (ISC98, HS94,CO99) \\
\hline 148 & 44 & $0057.7-7226$ & 1.4 & 3.2 & $5.73 \mathrm{e}-02 \pm 1.7 \mathrm{e}-03$ & 0.024 & SNR 0057-72.2 (YTK91,WW92) $13 \mathrm{~cm}$ \\
\hline 157 & 36 & $0053.7-7227$ & 20.9 & 2.3 & $2.74 \mathrm{e}-02 \pm 1.2 \mathrm{e}-03$ & 0.005 & AGN? Radio SMC B0053-7227 (FHW98) $13 \mathrm{~cm}$ jets \\
\hline 160 & 53 & 0103.8-7227 & 49.0 & 7.8 & $1.63 \mathrm{e}-02 \pm 1.0 \mathrm{e}-03$ & 0.011 & [hard] associated with DEM S128? (FHP00) \\
\hline 162 & 45 & $0059.1-7227$ & 44.5 & 2.3 & $2.67 \mathrm{e}-02 \pm 1.1 \mathrm{e}-03$ & 0.006 & hard] G star identification in SCC99 unlikely \\
\hline 163 & 53 & $0103.8-7227$ & 76.9 & 6.6 & $6.86 \mathrm{e}-03 \pm 6.7 \mathrm{e}-04$ & 0.011 & HMXB Be/X AXJ0105-722, pulsar (YK98b, FHP00) \\
\hline 170 & 40 & 0055.8-7229 & 14.2 & 3.6 & $5.48 \mathrm{e}-03 \pm 5.4 \mathrm{e}-04$ & 0.006 & [hard] [nonstar] \\
\hline 176 & 13 & $0035.3-7230$ & 6.1 & 1.2 & $4.40 \mathrm{e}-01 \pm 5.4 \mathrm{e}-03$ & 0.075 & SSS 1E0035.4-7230 (KP96) \\
\hline 185 & 42 & 0056.5-7233 & 80.6 & 19.0 & $2.32 \mathrm{e}-03 \pm 4.7 \mathrm{e}-04$ & 0.007 & \\
\hline 194 & 42 & 0056.5-7233 & 26.7 & 6.5 & $1.00 \mathrm{e}-02 \pm 7.2 \mathrm{e}-04$ & 0.007 & SNR 0056-72.5 (WW92) $13 \mathrm{~cm}$ \\
\hline 217 & 52 & 0103.2-7239 & 54.2 & 2.1 & $3.12 \mathrm{e}-01 \pm 4.6 \mathrm{e}-03$ & 0.056 & SNR 0103-72.6 (WW92, CSM97) $13 \mathrm{~cm}$ \\
\hline 220 & 47 & $0100.1-7240$ & 62.1 & 8.1 & $9.98 \mathrm{e}-03 \pm 9.5 \mathrm{e}-04$ & 0.007 & \\
\hline 227 & 55 & $0105.4-7240$ & 74.9 & 14.2 & $9.32 \mathrm{e}-04 \pm 1.3 \mathrm{e}-03$ & 0.002 & \\
\hline 230 & 55 & 0105.4-7240 & 51.4 & 2.4 & $1.20 \mathrm{e}-02 \pm 7.1 \mathrm{e}-04$ & 0.002 & [hard] \\
\hline 232 & 58 & 0107.1-7241 & 23.3 & 8.6 & $4.93 \mathrm{e}-03 \pm 5.6 \mathrm{e}-04$ & 0.004 & [hard] \\
\hline 234 & 39 & 0055.6-7241 & 20.5 & 3.4 & $8.73 \mathrm{e}-03 \pm 6.7 \mathrm{e}-04$ & 0.003 & \\
\hline 241 & 35 & $0053.2-7242$ & 20.5 & 3.5 & $3.68 \mathrm{e}-02 \pm 1.5 \mathrm{e}-03$ & 0.009 & HMXB Be/X XTEJ0055-724, pulsar (ML98, SCI98, SCB99, CO99) \\
\hline 242 & 34 & $0052.1-7242$ & 33.9 & 25.7 & $8.50 \mathrm{e}-02 \pm 4.7 \mathrm{e}-03$ & 0.008 & HMXB? XTEJ0053-724, pulsar (CML98) \\
\hline 252 & 59 & 0107.1-7244 & 67.8 & 13.7 & $5.62 \mathrm{e}-03 \pm 1.1 \mathrm{e}-03$ & 0.006 & \\
\hline 265 & 27 & $0050.1-7248$ & 26.4 & 45.8 & $5.45 \mathrm{e}-03 \pm 1.5 \mathrm{e}-03$ & 0.016 & HMXB Be/X RXJ0051.8-7231, pulsar (WW92, ISA97, SCB99) \\
\hline 279 & 56 & 0105.6-7251 & 20.3 & 11.1 & $7.44 \mathrm{e}-04 \pm 1.0 \mathrm{e}-03$ & 0.007 & \\
\hline 285 & 30 & 0050.9-7253 & 50.9 & 12.7 & $6.86 \mathrm{e}-03 \pm 8.7 \mathrm{e}-04$ & 0.012 & SNR 0050-72.8 (WW92) $13 \mathrm{~cm}$ \\
\hline 295 & 19 & 0045.6-7256 & 54.1 & 49.8 & $4.06 \mathrm{e}-03 \pm 5.1 \mathrm{e}-03$ & 0.006 & AGN? Radio SMC B0045-7255 (FHW98) $13 \mathrm{~cm}$ \\
\hline 320 & 48 & 0101.3-7300 & 53.7 & 17.2 & $3.58 \mathrm{e}-03 \pm 7.9 \mathrm{e}-04$ & 0.027 & [fg Star] GSC 9141.7584 \\
\hline 333 & 31 & $0051.1-7304$ & 79.2 & 12.5 & $1.21 \mathrm{e}-03 \pm 4.0 \mathrm{e}-04$ & 0.008 & \\
\hline 361 & 72 & 0102.3-7318 & 8.6 & 32.4 & $4.40 \mathrm{e}-02 \pm 9.6 \mathrm{e}-03$ & & SSS? RXJ0103.8-7254 (KP96) \\
\hline 379 & 62 & $0115.1-7315$ & 21.5 & 14.0 & $6.04 \mathrm{e}-03 \pm 8.2 \mathrm{e}-04$ & 0.005 & AGN $z=0.253($ TDZ97) \\
\hline 385 & 66 & 0118.1-7316 & 19.5 & 13.2 & $3.13 \mathrm{e}-02 \pm 1.6 \mathrm{e}-03$ & 0.013 & Cluster $z=0.0656$ (CGC97) \\
\hline 413 & 16 & $0045.5-7325$ & 50.6 & 6.8 & $2.98 \mathrm{e}-02 \pm 1.6 \mathrm{e}-03$ & 0.020 & SNR 0045-73.4 (WW92, RLG94) $13 \mathrm{~cm}$ \\
\hline 414 & 15 & $0044.7-7324$ & 43.7 & 8.5 & $1.73 \mathrm{e}-02 \pm 1.3 \mathrm{e}-03$ & 0.007 & SNR 0044-7325 (WW92, RLG94) $13 \mathrm{~cm}$ \\
\hline 419 & 16 & 0045.5-7325 & 88.2 & 4.7 & $3.72 \mathrm{e}-02 \pm 1.5 \mathrm{e}-03$ & 0.020 & [SNR] $13 \mathrm{~cm}$, part of source $413 ?$ \\
\hline 424 & 25 & $0050.0-7327$ & 36.8 & 2.2 & $1.46 \mathrm{e}-02 \pm 8.6 \mathrm{e}-04$ & 0.011 & [hard] \\
\hline 431 & 64 & $0116.1-7326$ & 67.5 & 5.9 & $5.51 \mathrm{e}-03 \pm 5.5 \mathrm{e}-04$ & 0.005 & [stellar] GSC 9142.1764 \\
\hline 434 & 18 & 0045.6-7329 & 37.8 & 4.0 & $1.41 \mathrm{e}-02 \pm 9.6 \mathrm{e}-04$ & 0.006 & [hard] \\
\hline 437 & 22 & 0047.3-7330 & 2.4 & 4.7 & $1.77 \mathrm{e}-02 \pm 1.1 \mathrm{e}-03$ & 0.007 & SNR 0047-73.5 (WW92, FPH00) $13 \mathrm{~cm}$ \\
\hline 453 & 29 & 0050.3-7335 & 36.0 & 1.9 & $2.15 \mathrm{e}-02 \pm 1.0 \mathrm{e}-03$ & 0.009 & HMXB Be/X RXJ0052.1-7319, pulsar (LPM99, ISC99) \\
\hline 454 & 21 & 0046.6-7335 & 34.0 & 10.8 & $5.15 \mathrm{e}-03 \pm 7.2 \mathrm{e}-04$ & 0.004 & SNR 0046-73.5 (WW92) $13 \mathrm{~cm}$ \\
\hline 461 & 24 & $0049.2-7337$ & 26.1 & 2.0 & $1.41 \mathrm{e}-01 \pm 2.7 \mathrm{e}-03$ & 0.060 & SNR 0049-73.6 (WW92, CSM97) $13 \mathrm{~cm}$ \\
\hline 478 & 65 & $0117.2-7341$ & 16.2 & 0.8 & $1.11 \mathrm{e}-01 \pm 1.8 \mathrm{e}-03$ & 0.016 & fg Star G5V, HD 8191 (WW92, CSM97) \\
\hline 480 & 14 & $0043.7-7343$ & 65.9 & 16.7 & $1.19 \mathrm{e}-03 \pm 1.6 \mathrm{e}-03$ & 0.004 & \\
\hline 482 & 63 & 0115.8-7342 & 15.9 & 0.6 & $5.82 \pm 1.2 \mathrm{e}-02$ & 0.069 & HMXB supergiant SMC X-1, pulsar (WW92) \\
\hline 496 & 28 & $0050.1-7346$ & 43.5 & 10.4 & $9.39 \mathrm{e}-03 \pm 2.4 \mathrm{e}-03$ & 0.010 & [nonstar] \\
\hline 623 & 33 & $0051.4-7455$ & 2.5 & 0.7 & $4.12 \pm 8.4 \mathrm{e}-03$ & 0.413 & fg Star G3:V+, RSCVn type, HD 5303 (WW92), $13 \mathrm{~cm}$ \\
\hline
\end{tabular}

Notes to the remark column to this and following tables:

Abbreviations for references given in parenthesis are described in the literature list.

Unsecure identifications from positional uncertainty begin with? in the remark.

Candidates from literature are marked with? after source class name.

Abbreviations: GSC: HST guide star catalogue (Lasker et al. 1990); fg: foreground.

The majority of classified sources (Table 7) consists of AGN and AGN candidates (46). Six AGN were optically identified by Tinney et al. (1997) and we suggest 22 new candidates which have an unresolved radio counterpart (mainly at $\lambda=13 \mathrm{~cm}$ ). We classify as AGN additional 12 sources with high X-ray to optical flux ratio which are located south of the main body of the SMC. Table 7 contains further six uncertain AGN candidates due to large position uncertainties. The highly extended source 385 is identified with a cluster of galaxies. Another 24 sources are identified (12) and classified (12) as foreground stars.

$\mathrm{X}$-ray sources intrinsic to the SMC are HMXBs, SSSs and SNRs. Fifteen HMXB and candidates, mainly $\mathrm{X}$-ray pulsars discovered with instruments sensitive at energies of $1-10 \mathrm{keV}$ are included. Only three pulsars compiled in Table 4 of KPFH99 (namely AX J0051-722, XTE J0054-720 and XTEJ0111.2-7317) were not detected in PSPC data. We note that source 79 in KPFH99 
Table 4. PSPC sources detected in the ROSAT all-sky survey

\begin{tabular}{|c|c|c|c|c|c|c|c|}
\hline 1 & 2 & 3 & 4 & 5 & 6 & 7 & 8 \\
\hline No & $\begin{array}{l}\text { BSC name } \\
\text { 1RXS }\end{array}$ & $\begin{array}{c}d \\
{\left[{ }^{\prime \prime}\right]}\end{array}$ & $\begin{array}{c}r_{90} \\
{\left[{ }^{\prime \prime}\right]}\end{array}$ & $\begin{array}{r}r_{\mathrm{BSC}} \\
{\left[{ }^{\prime \prime}\right]}\end{array}$ & $\begin{array}{r}\text { PSPC Count Rate } \\
{\left[\operatorname{cts~s}^{-1}\right]}\end{array}$ & $\begin{array}{l}\text { BSC Rate } \\
{\left[\operatorname{cts~s}^{-1}\right]}\end{array}$ & Remarks \\
\hline 8 & 1RXS J005052.4-710914 & 10.5 & 20.2 & 11 & $2.04 \mathrm{e}-01 \pm 1.1 \mathrm{e}-02$ & $1.20 \mathrm{e}-01 \pm 2.3 \mathrm{e}-02$ & fg Star F5V, HD 5028 (WW92) \\
\hline 47 & 1RXS J005837.9-713542 & 8.8 & 1.3 & 9 & $3.68 \mathrm{e}-01 \pm 4.9 \mathrm{e}-03$ & $2.81 \mathrm{e}-01 \pm 3.2 \mathrm{e}-02$ & SSS 1E0056.8-7154, PN LIN333 (KP96) \\
\hline 52 & 1RXS J003704.1-713821 & 34.1 & 17.5 & 19 & $3.86 \mathrm{e}-02 \pm 2.8 \mathrm{e}-03$ & $5.71 \mathrm{e}-02 \pm 1.4 \mathrm{e}-02$ & $\begin{array}{l}\text { AGN? [hard] [nonstar] Radio SMC } \\
\text { B0034-7155 (FJW97) } 13 \mathrm{~cm} \text { jets }\end{array}$ \\
\hline 107 & 1RXS J010403.5-720158 & 11.2 & 0.7 & 7 & $2.24 \pm 2.1 \mathrm{e}-02$ & $2.02 \pm 7.7 \mathrm{e}-02$ & SNR 0102-72.3 (WW92, FPW99) $13 \mathrm{~cm}$ \\
\hline 217 & 1RXS J010500.1-722305 & 17.5 & 2.1 & 11 & $3.12 \mathrm{e}-01 \pm 4.6 \mathrm{e}-03$ & $2.36 \mathrm{e}-01 \pm 2.8 \mathrm{e}-02$ & SNR 0103-72.6 (WW92, CSM97) $13 \mathrm{~cm}$ \\
\hline 461 & 1RXS J005108.0-732134 & 9.8 & 2.0 & 21 & $1.41 \mathrm{e}-01 \pm 2.7 \mathrm{e}-03$ & $8.08 \mathrm{e}-02 \pm 1.6 \mathrm{e}-02$ & SNR 0049-73.6 (WW92, CSM97) $13 \mathrm{~cm}$ \\
\hline 478 & 1RXS J011836.8-732529 & 6.2 & 0.8 & 14 & $1.11 \mathrm{e}-01 \pm 1.8 \mathrm{e}-03$ & $6.45 \mathrm{e}-02 \pm 1.8 \mathrm{e}-02$ & fg Star G5V, HD 8191 (WW92, CSM97) \\
\hline 482 & 1RXS J011705.9-732632 & 1.5 & 0.6 & 8 & $5.82 \pm 1.2 \mathrm{e}-02$ & $7.20 \mathrm{e}-01 \pm 6.4 \mathrm{e}-02$ & $\begin{array}{l}\text { HMXB supergiant SMC X-1, pulsar } \\
\text { (WW92) }\end{array}$ \\
\hline 512 & 1RXS J004820.7-733154 & 14.9 & 24.1 & 8 & $8.10 \mathrm{e}-03 \pm 2.5 \mathrm{e}-03$ & $1.99 \mathrm{e}-01 \pm 2.2 \mathrm{e}-02$ & $\begin{array}{l}\text { SSS RXJ0048.4-7332, symbiotic star M0 } \\
\text { (KP96, M92) }\end{array}$ \\
\hline 621 & 1RXS J004402.7-743737 & 6.1 & 5.9 & 10 & $8.96 \mathrm{e}-02 \pm 1.6 \mathrm{e}-03$ & $6.51 \mathrm{e}-02 \pm 1.3 \mathrm{e}-02$ & [fg Star] GSC 9141.1004 \\
\hline 623 & 1RXS J005307.5-743903 & 2.4 & 0.7 & 6 & $4.12 \pm 8.4 \mathrm{e}-03$ & $2.38 \pm 6.3 \mathrm{e}-02$ & $\begin{array}{l}\text { fg Star G3:V+, RSCVn type, HD } 5303 \\
\text { (WW92), } 13 \mathrm{~cm}\end{array}$ \\
\hline 683 & 1RXS J012522.3-750015 & 2.1 & 10.2 & 12 & $6.26 \mathrm{e}-02 \pm 5.2 \mathrm{e}-03$ & $6.42 \mathrm{e}-02 \pm 1.3 \mathrm{e}-02$ & fg Star SAO 255776 (SIM) \\
\hline 709 & 1RXS J005655.1-751349 & 2.6 & 4.3 & 7 & $2.13 \mathrm{e}-01 \pm 2.4 \mathrm{e}-03$ & $3.00 \mathrm{e}-01 \pm 2.4 \mathrm{e}-02$ & [AGN] GSC 9142.0531 \\
\hline 721 & 1RXS J012254.1-752117 & 10.2 & 1.4 & 9 & $8.15 \mathrm{e}-01 \pm 1.2 \mathrm{e}-02$ & $5.28 \mathrm{e}-01 \pm 6.7 \mathrm{e}-02$ & fg Star WD, FAUST 199 (SIM) \\
\hline 734 & 1RXS J013114.0-755643 & 15.0 & 17.7 & 7 & $2.28 \mathrm{e}-01 \pm 9.7 \mathrm{e}-03$ & $2.27 \mathrm{e}-01 \pm 2.0 \mathrm{e}-02$ & fg Star K0, CD-76 56 (SIM) \\
\hline
\end{tabular}

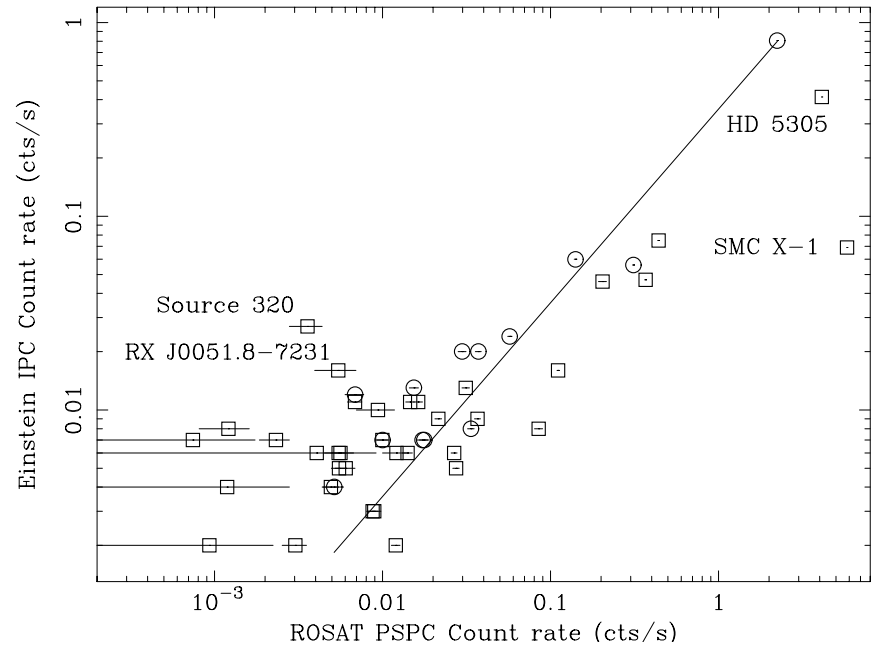

Fig. 4. Einstein IPC vs. ROSAT PSPC count rates. Sources identified with SNRs are marked with circles. The line is a linear fit to the SNR data points

(source 188 in Table 7 ) is probably not associated with AX J0051-722 because of the large $\left(3^{\prime}\right)$ offset in position. Most of the X-ray binary systems were detected with PSPC count rates between $2.710^{-3}$ cts s $^{-1}$ and $8.910^{-2}$ cts $\mathrm{s}^{-1}$ which converts to X-ray luminosities between $4.510^{34} \mathrm{erg} \mathrm{s}^{-1}$ and $1.510^{36} \mathrm{erg} \mathrm{s}^{-1}$ (using a typical conversion factor of $1.6710^{37} \mathrm{erg} \mathrm{s}^{-1} / \mathrm{cts} \mathrm{s}^{-1}$; Kahabka \& Pietsch 1996, hereafter KP96). This "quiescent" level is similar to that seen from persistent galactic high mass X-ray binaries like e.g. X Persei, RX J0146.9-6121 or RX J1037.5-5647 (Haberl et al. 1998; Reig \& Roche 1999). With the exception of SMCX-1 and the peculiar soft X-ray pulsar RX J0059.2-7138 no other $\mathrm{X}$-ray binary was found with a luminosity in excess of

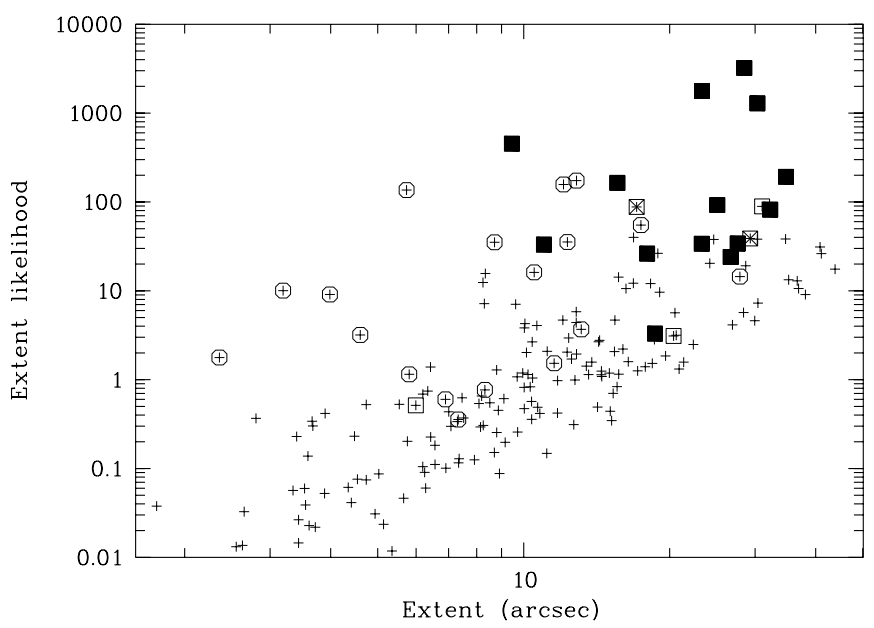

Fig. 5. Source extent and extent likelihood for the SMC PSPC sources with off-axis angle less than $18^{\prime}$ (plus signs). SNRs are marked with squares (filled: secure, open: candidates, crossed: classified in this work) and known point sources (X-ray binaries, SSSs, stars and AGN) with hexagons

$10^{38} \mathrm{erg} \mathrm{s}^{-1}$ in the ROSAT band $(0.1-2.4 \mathrm{keV})$. This casts doubt on earlier suggestions made before the launch of ROSAT (when only few X-ray binary systems were known in the MCs) that the fraction of high luminosity sources emitting near the Eddington limit in the MCs is higher than in the galactic population (Pakull 1989).

Four sources were earlier identified as SSSs, two were suggested by KP96 and three more are classified here as SSSs. Source 691 is likely identified with the SMC symbiotic star LIN 358 and is located together with source 664 remarkably far south of the SMC main body. Source 16 as third classified SSS is found north of the main body of the SMC. 
Table 5. PSPC sources from the catalogue of Kahabka et al. (1999) not detected in the present analysis

\begin{tabular}{|c|c|c|}
\hline \multicolumn{2}{|c|}{ KРFH99 } & \multirow{2}{*}{ Comment $^{1}$} \\
\hline No & Name & \\
\hline 6 & $0035.0-7354$ & $\mathrm{D}$ \\
\hline 14 & $0036.0-7210$ & $\mathrm{ML}_{\mathrm{exi}}=11$ \\
\hline 24 & $0038.0-7344$ & $\mathrm{ML}_{\text {exi }}=14$ \\
\hline 25 & 0038.0-7327 & near WSS, may be real \\
\hline 38 & $0042.2-7338$ & $\mathrm{D}$ \\
\hline 60 & 0047.5-7308 & two catalogue sources $(413 / 419)$ \\
\hline 73 & $0050.7-7226$ & $\mathrm{D}$ \\
\hline 75 & $0050.8-7341$ & WSS, not real \\
\hline 81 & $0051.7-7341$ & $\mathrm{ML}_{\mathrm{exi}}=10$ \\
\hline 90 & 0053.0-7239 & $\mathrm{ML}_{\text {exi }}=10$ \\
\hline 91 & $0053.1-7311$ & $\mathrm{ML}_{\text {exi }}=11$ \\
\hline 97 & $0053.8-7252$ & $\mathrm{ML}_{\text {exi }}=11$ \\
\hline 99 & $0054.3-7330$ & $\mathrm{ML}_{\text {exi }}=11$ \\
\hline 112 & $0055.9-7257$ & $\mathrm{D}$ \\
\hline 113 & $0056.4-7159$ & $\mathrm{D}$ \\
\hline 119 & $0057.2-7156$ & $\mathrm{ML}_{\text {exi }}=13$ \\
\hline 132 & 0058.5-7249 & $\mathrm{D}$ \\
\hline 136 & $0058.9-7255$ & $\mathrm{D}$ \\
\hline 142 & $0059.7-7148$ & $\mathrm{ML}_{\mathrm{exi}}=12$ \\
\hline 156 & 0101.0-7151 & $\mathrm{ML}_{\mathrm{exi}}=11$ \\
\hline 157 & 0101.0-7211 & $\mathrm{D}$ \\
\hline 161 & 0101.3-7118 & $\mathrm{D}$ \\
\hline 165 & 0101.8-7249 & WSS, not real \\
\hline 167 & $0101.8-7233$ & $\mathrm{ML}_{\mathrm{exi}}=14$ \\
\hline 168 & $0102.1-7236$ & $\mathrm{D}$ \\
\hline 172 & $0102.8-7216$ & $\mathrm{D}$ \\
\hline 173 & 0102.9-7111 & $\mathrm{D}$ \\
\hline 185 & 0104.2-7102 & WSS, not real \\
\hline 186 & $0104.7-7203$ & near WSS, may be real \\
\hline 190 & 0105.3-7126 & $\mathrm{ML}_{\mathrm{exi}}=14$ \\
\hline 192 & 0105.4-7219 & $\mathrm{ML}_{\mathrm{exi}}=11$ \\
\hline 197 & $0106.3-7125$ & $\mathrm{D}$ \\
\hline 207 & $0108.5-7247$ & $\mathrm{ML}_{\mathrm{exi}}=12$ \\
\hline 210 & 0109.0-7243 & $\mathrm{ML}_{\text {exi }}=13$ \\
\hline 213 & 0109.5-7226 & $\mathrm{ML}_{\text {exi }}=10$ \\
\hline 215 & 0110.6-7217 & $\mathrm{ML}_{\mathrm{exi}}=12$ \\
\hline 223 & $0112.7-7207$ & not real, largest extent in KPFH99 \\
\hline 241 & $0116.8-7314$ & $\mathrm{D}$ \\
\hline 243 & $0117.2-7238$ & $\mathrm{D}$ \\
\hline
\end{tabular}

1 Artifacts marked as D by KPFH99; likelihood of existence $\mathrm{ML}_{\text {exi }}$ from KPFH99.

SNRs form the largest group of the SMC sources in Table 7. However, it should be noted that the class of SNRs is more complete than the class of X-ray binaries because the latter can not be uniquely separated from the hard sources. Fourteen clearly extended X-ray sources are identified with known SNRs and three with new candidates from radio investigations. In the work here another two sources are classified as SNR, both significantly extended X-ray sources. SNR 0102-72.3 (source 107) is by far the brightest in this sample of SNRs and amounts to about $75 \%$ of the total known SNR X-ray luminosity in

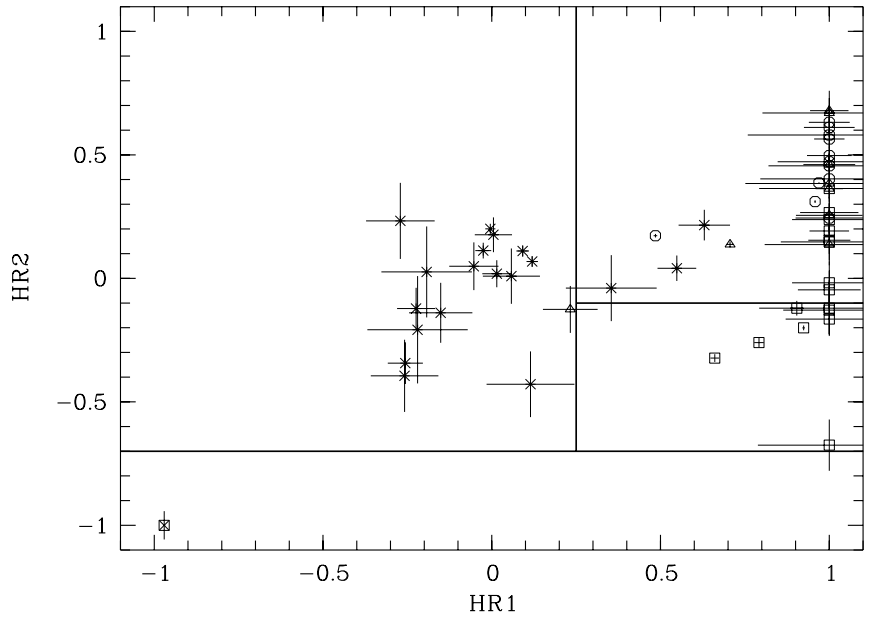

Fig. 6. Hardness ratios of identified and classified PSPC sources in the SMC area. X-ray binaries are marked with a hexagon, SSSs with crossed square, SNRs with square, stars with $\times$, and AGN with triangle. The thick lines separate areas where only members of a single class were found in the LMC (HP99). Only sources with error on HR1 and HR2 less than 0.25 are shown

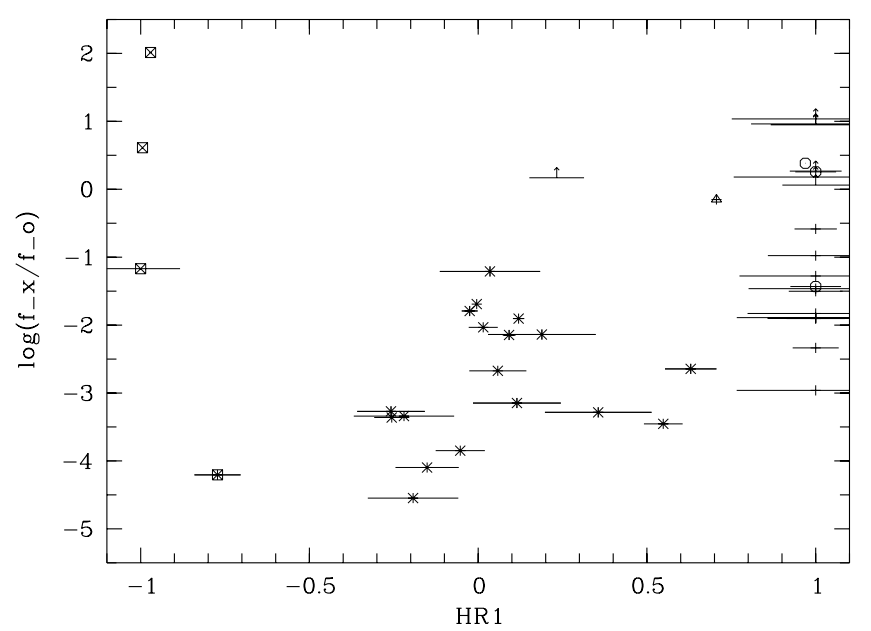

Fig. 7. Flux ratio $f_{\mathrm{x}} / f_{\mathrm{opt}}$ as function of hardness ratio 1 (HR1). The optically identified sources are marked with different symbols (X-ray binaries: hexagon, SSSs: crossed square, foreground stars: $\times$ and AGN: triangle). Unidentified sources with error on HR1 less than 0.25 and nearby GSC entry are shown with HR1 error bars. Some lower limits derived from the optically brightest objects in the error circle are indicated by arrows

the SMC of $\sim 510^{37} \mathrm{erg} \mathrm{s}^{-1}$ (using the same count rate conversion factor from above).

The classification of sources as [hard], [nonstar] and [stellar] does not allow a unique identification of the origin of the X-ray emission but restricts the number of possibilities. [Hard] and [nonstar] objects are likely candidates for X-ray binaries or background AGN while the [stellar] classification most likely selects foreground stars, but bright SMC stars can not be excluded as e.g. the Be X-ray binaries show typical optical magnitudes of 15 (cf. HP99). 


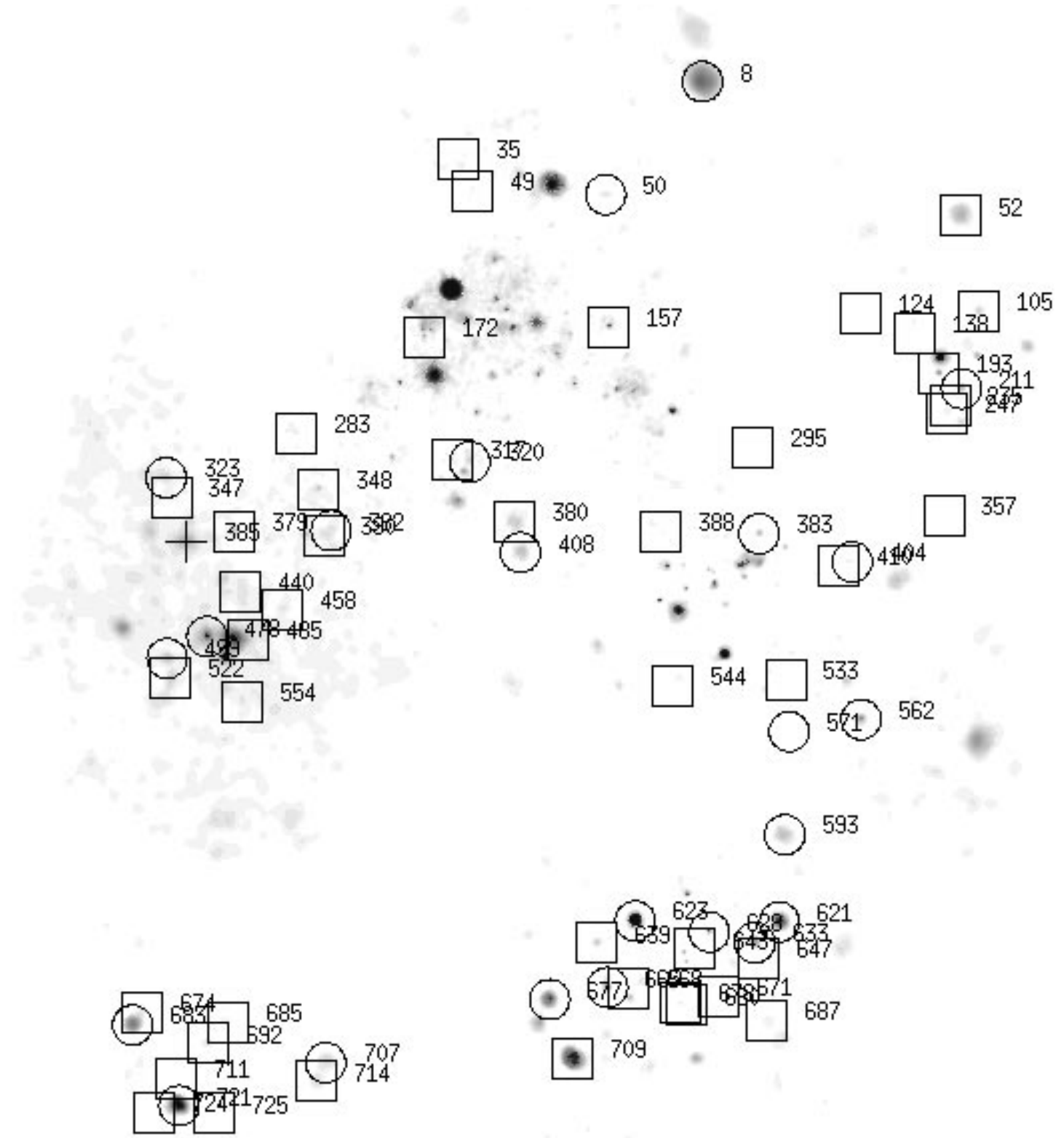

729

734 735

Fig. 8. Distribution of foreground and background objects in the SMC region detected with the ROSAT PSPC. Foreground stars and candidates are marked with circle, AGN and AGN candidates with squares. The only source (385) identified with a cluster of galaxies is marked by a plus. Source numbers refer to Table 7

\subsection{Radio sources}

Besides the radio catalogues covered by SIMBAD we correlated the PSPC catalogue with a preliminary list of radio sources resulting from the ATCA mosaic high resolution survey (Filipović \& Stavely-Smith, in preparation). The PSPC sources detected at $\lambda=13 \mathrm{~cm}(\nu=2.37 \mathrm{GHz})$ are indicated by " $13 \mathrm{~cm}$ " in the comment column of Table 7 . An inspection of the source morphologies on the $13 \mathrm{~cm}$ image revealed many point sources which are candidates for background AGN. In three cases jet like structures indicate radio lobes (sources 52, 157 and 390). Most known SNRs and SNR candidates are detected in the ATCA $\lambda=13 \mathrm{~cm}$ image and a new candidate (source 448) is found in the Eastern Wing of the SMC. 


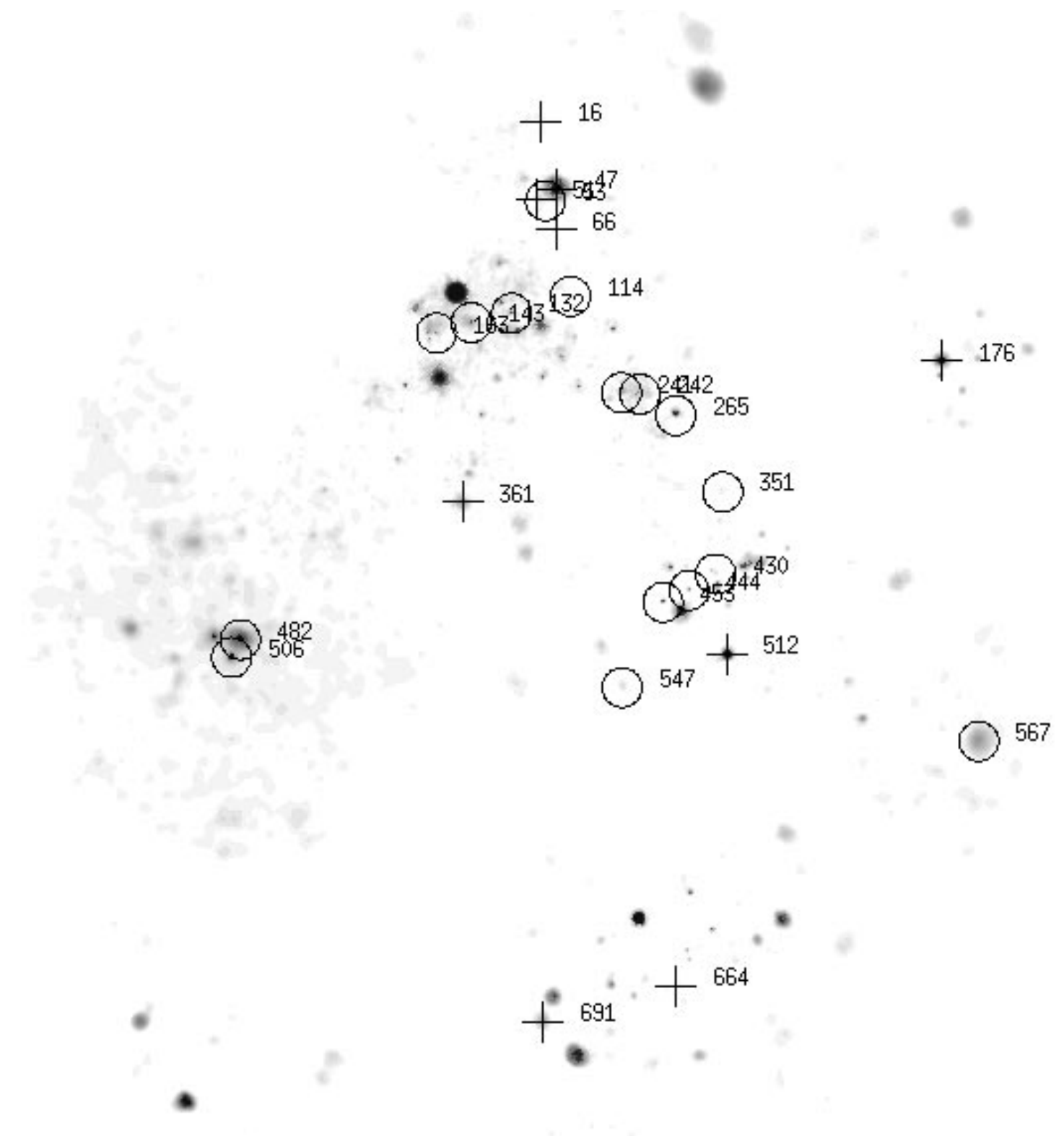

Fig. 9. Distribution of SSS (plus) and X-ray binaries (circle) including new candidates from this work

\subsection{Spatial distribution of source populations}

The spatial distribution of the different identified (including elsewhere published candidates) and classified PSPC sources from our SMC catalogue is shown in Figs. 8-10. Foreground stars and AGN are found in the whole field covered by PSPC observations (Fig. 8). Although, a lower density of detected AGN is expected along the main body of the SMC due to absorption of the X-ray emission, the small number of background objects in that area seen in
Fig. 8 is mainly caused by incomplete classification of hard $\mathrm{X}$-ray sources. The SMC intrinsic sources like HMXBs and SNRs are preferentially distributed along the main body of the SMC (Figs. 9 and 10). The high density might be caused by projection of a more extended distribution as Cepheid distances indicate a depth of the SMC of about $20 \mathrm{kpc}$ (Mathewson et al. 1988). SSSs are detected around the main body (Fig. 9), very similar to the LMC where the SSSs are only found around the optical bar (HP99). SSSs may also exist inside the main SMC body but their 


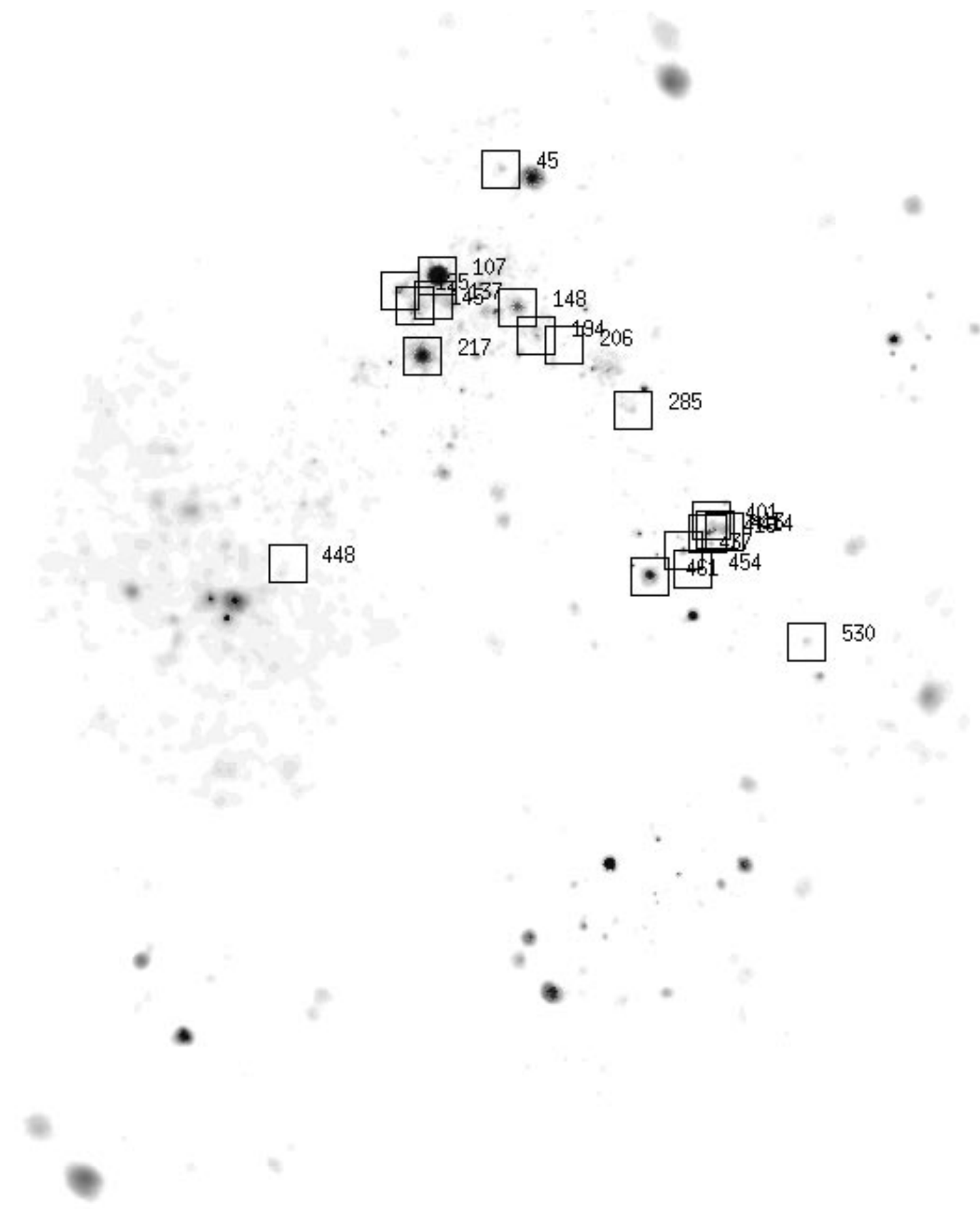

Fig. 10. Distribution of SNRs and SNR candidates in the SMC

supersoft X-ray emission is absorbed by the dense gas and dust.

\section{Summary}

We analyzed the complete ROSAT PSPC pointed data covering the MCs to obtain a comprehensive overview of the X-ray source content of these nearby galaxies. The source catalogue of the LMC was published by Haberl \& Pietsch (1999) and in the current work a complementary catalogue of 517 discrete sources in the SMC is presented. About $30 \%$ of the PSPC sources are identified with known sources or at least classified as candidates for AGN, foreground stars, SSSs or SNRs. A high fraction of AGN and AGN candidates was found mainly from comparison with available radio data. These samples of different source classes allow to investigate their properties on a large statistical basis in a galaxy as a whole, but further identification work is required to confirm the classification and further enlarge the samples. 
Table 6. Classification criteria

\begin{tabular}{|c|c|c|c|}
\hline Source class & selection & unidentified & $\begin{array}{r}\text { classified } \\
\text { sources }\end{array}$ \\
\hline SSS & HR $1+$ EHR $1<-0.70$ & 3 & 3 \\
\hline \multirow[t]{4}{*}{ fg star } & HR1+EHR $1<0.25$, HR2 - EHR2 $>-0.70$ & 7 & \\
\hline & $\mathrm{HR} 1<0.25, \mathrm{HR} 2>-0.70, \mathrm{EHR} 1<0.25, \mathrm{EHR} 2<0.25$ & 1 & \\
\hline & EHR $1<0.25,-0.75<\mathrm{HR} 1<0.25, \log \left(f_{\mathrm{x}} / f_{\mathrm{opt}}\right)<-0.5$ & 2 & \\
\hline & EHR $1<0.25,0.25<\mathrm{HR} 1<0.75, \log \left(f_{\mathrm{x}} / f_{\text {opt }}\right)<-1.0$ & 3 & 12 \\
\hline \multirow[t]{3}{*}{ SNR } & $\mathrm{ML}_{\text {ext }}>50$, offaxis angle $<18^{\prime}$ & 1 & \\
\hline & $\mathrm{HR} 1-\mathrm{R} 1>0.25, \mathrm{HR} 2-\mathrm{EHR} 2>-0.70, \mathrm{HR} 2+\mathrm{EHR} 2<-0.10$ & 1 & \\
\hline & $\mathrm{HR} 1>0.25,-0.70<\mathrm{HR} 2<-0.10, \mathrm{EHR} 1<0.25$, EHR $2<0.25$ & 0 & 2 \\
\hline \multirow[t]{2}{*}{ hard } & HR $1-$ EHR $1>0.75$, HR $2-$ EHR $2>-0.10$ & 25 & \\
\hline & $\mathrm{HR} 1>0.75, \mathrm{HR} 2>-0.10, \mathrm{EHR} 1<0.25, \mathrm{EHR} 2<0.25$ & 1 & 26 \\
\hline \multirow[t]{2}{*}{ AGN } & EHR $1<0.25, \mathrm{HR} 1>0.25, \log \left(f_{\mathrm{x}} / f_{\text {opt }}\right)>-1.0$ & 1 & \\
\hline & nonstar south of $-74^{\circ} 30^{\prime}$ & 12 & 13 \\
\hline stellar & $r_{90}<20^{\prime \prime}$, distance to GSC entry $<r_{90}$ & 16 & 9 \\
\hline nonstar & lower limit of $\log \left(f_{\mathrm{x}} / f_{\mathrm{opt}}\right)>0.0$ & 31 & 31 \\
\hline
\end{tabular}

Acknowledgements. The ROSAT project is supported by the German Bundesministerium für Bildung und Forschung (BMBF/DLR) and the Max-Planck-Gesellschaft. This research has made extensive use of the SIMBAD data base operated at CDS, Strasbourg, France. For the presentation of Fig. 1 we used the Karma software package developed at ATNF.

\section{References}

Bickert K.F., Greiner J., Stencel R.E., 1996, LNP 472, Supersoft X-ray Sources, Greiner J. (ed.), p. 225 (BGS96)

Clark G.W., Remillard R.A., Woo J.W., 1997, ApJ 474, L111 (CRW97)

Coe M.J., Orosz J.A., 1999, MNRAS (in press) astro-ph/9908303, (CO99)

Corbet R., Marshall F.E., Lochner J.C., Ozaki M., Ueda Y., 1997, IAU Circ. 6803 (CML98)

Cowley A.P., Schmidtke P.C., McGrath T.K., Ponder A.L., Fertig R.M., 1997, PASP 109, 21 (CSM97)

Crampton D., Gussie G., Cowley A.P., Schmidtke P.C., 1997, AJ 114, 2353 (CGC97)

Filipović M.D., Jones P.A., White G.L., Haynes R.F., Klein U., Wielebinski R., 1997, A\&AS 121, 321 (FJW97)

Filipović M.D., Haberl F., Pietsch W., Morgan D., 2000, A\&A (submitted) (FHP00)

Filipović M.D., Haynes R.F., White G.L., Jones P.A., 1998, A\&AS 130, 421 (FHW98)

Filipović M.D., Pietsch W., Haberl F., 2000, A\&A (submitted) (FPH00)

Filipović M.D., Pietsch W., Haynes R.F., et al., 1998, A\&AS 127, 119 (FPH98)

Filipović M.D., Pietsch W., White G.L., Haberl F., StavelySmith L., Jones P.A., Haynes R.F., Sasaki M., 1999, IAU Symp. 192, 104 (FPW99)

Haberl F., Angelini L., Motch C., White N.E., 1998, A\&A 330, 189

Haberl F., Pietsch W., 1999, A\&AS 139, 277 (HP99)

Hughes J.P., 1994, ApJ 427, L25 (H94)
Hughes J.P., Smith R.C., 1994, AJ 107, 1363 (HS94)

Imanishi K., Yogokawa J., Koyama K., 1998, IAU Circ. 7040 (IYK98)

Israel G.L., Stella L., Angelini L., et al., 1997, ApJ 484, L141 (ISA97)

Israel G.L., Stella L., Campana S., Covino S., Ricci D., Oosterbroek T., 1998, IAU Circ. 6999 (ISC98)

Israel G.L., Stella L., Covino S., Campana S., Mereghetti S., 1999, IAU Circ. 7101 (ISC99)

Kahabka P., Pietsch W., Hasinger G., 1994, A\&A 288, 538

Kahabka P., Pietsch W., 1996, A\&A 312, 919 (KP96)

Kahabka P., Pietsch W., 1998, IAU Circ. 6840 (KP98)

Kahabka P., Pietsch W., Filipović M.D., Haberl F., 1999, A\&AS 136, 1 (KPFH99)

Lamb R.C., Prince T.A., Macomb D.J., Finger M.H., 1999, IAU Circ. 7081 (LPM99)

Lasker B.M., Sturch C.R., McLean B.J., Russel J.L., Jenkner H., Shara M.M., 1990, AJ 99, 2019

Macomb D.J., Finger M.H., Harmon B.A., Lamb R.C., Prince T.A., 1999, ApJ 518, L99 (MFH99)

Marshall F.E., Lochner J.C., 1998, IAU Circ. 6818 (ML98)

Mathewson D.S., Ford V.L., Visvanathan N., 1988, ApJ 333, 617

Pakull M., 1989, in Recent Developments of Magellanic Cloud Research, Boer K.S., Spite F., Stasinska G. (eds.). Paris: Observatoire de Paris, p. 183

Pfeffermann E., Briel U.G., Hippmann H., et al., 1986, Proc. SPIE 733, 519

Reig P., Roche P., 1999, MNRAS 306, 100

Rosado M., Le Coarer E., Georgelin Y.P., 1994, A\&A 286, 231 (RLG94)

Santangelo A., Cusumano G., Israel G.L., et al., 1998, IAU Circ. 6818 (SCI98)

Schmidtke P.C., Cowley A.P., 1998, IAU Circ. 6880 (SC98)

Schmidtke P.C., Cowley A.P., Crane J.D., et al., 1999, AJ 117, 927 (SCC99)

Southwell K.A., Charles P.A., 1996, MNRAS 281, L63 (SC96)

Stevens J.B., Coe M.J., Buckley D.A.H., 1999, MNRAS 309, 421 (SCB99)

Tinney C.G., Da Costa G.S., Zinnecker H., 1997, MNRAS 285, 111 (TDZ97) 
Trümper J., 1982, Adv. Space Res. 2, 241

Vogel M., Nussbaumer H., 1995, A\&A 301, 170 (VN95)

Voges W., Aschenbach B., Boller Th., et al., 1996, IAU Circ. 6420

Voges W., Aschenbach B., Boller Th., et al., 1999, A\&A 349, 389

Wang Q., Hamilton T.T., Helfand D.J., Wu X., 1991, ApJ 374, 475

Wang Q., Wu X., 1992, ApJS 78, 391 (WW92)
Ye T., Turtle A.J., Kennicutt R.C. Jr., 1991, MNRAS 249, 722 (YTK91)

Yogokawa J., Koyama K., 1998, IAU Circ. 6853 (YK98a)

Yogokawa J., Koyama K., 1998, IAU Circ. 7028 (YK98b)

Yogokawa J., Koyama K., 1998, IAU Circ. 6835 (YK98c)

Zimmermann H.U., Becker W., Belloni T., Döbereiner S., Izzo C., Kahabka P., Schwentker O., 1994, EXSAS User's Guide, MPE report 257 
Table 7. Identified and classified PSPC sources

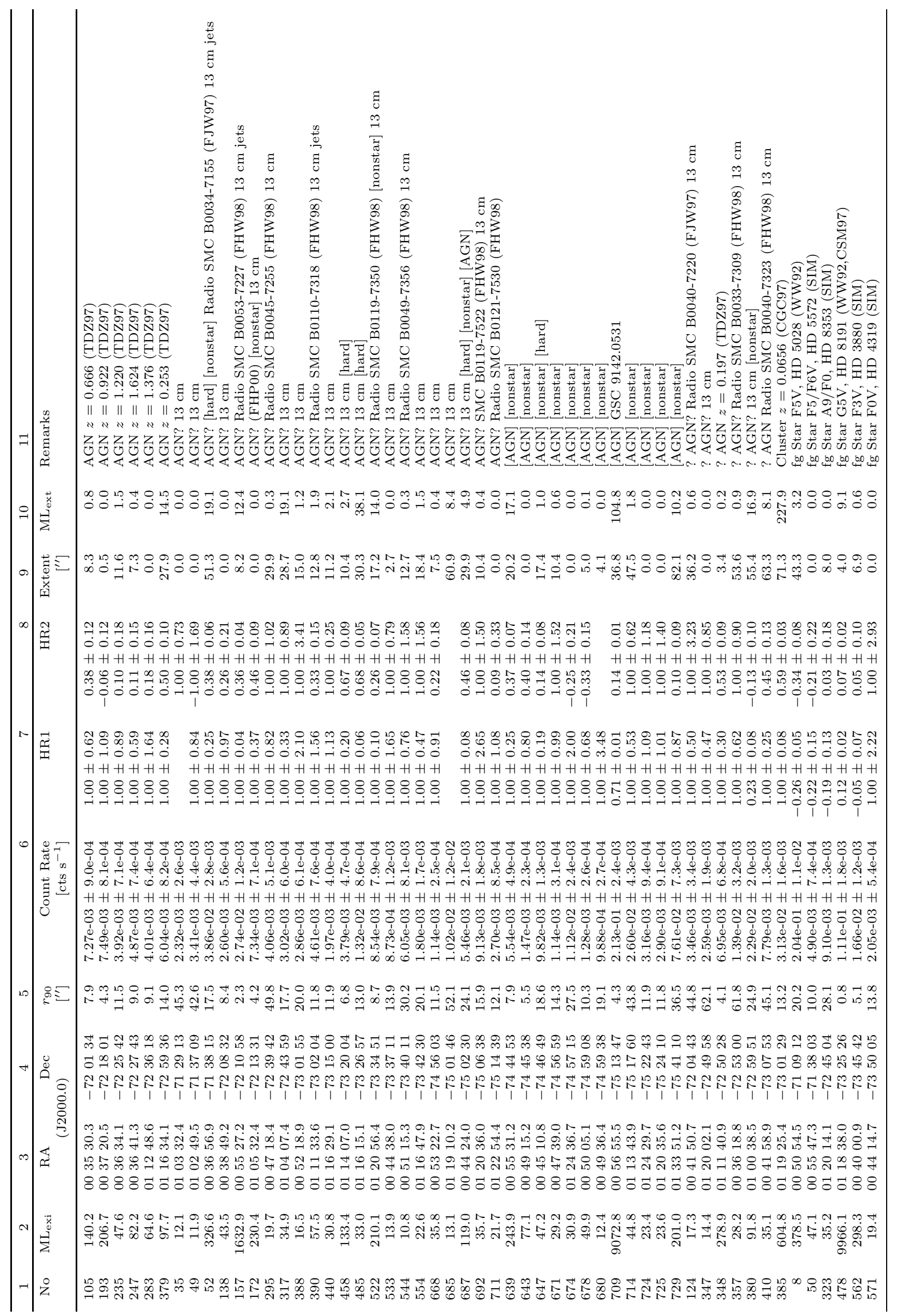


Table 7. continued

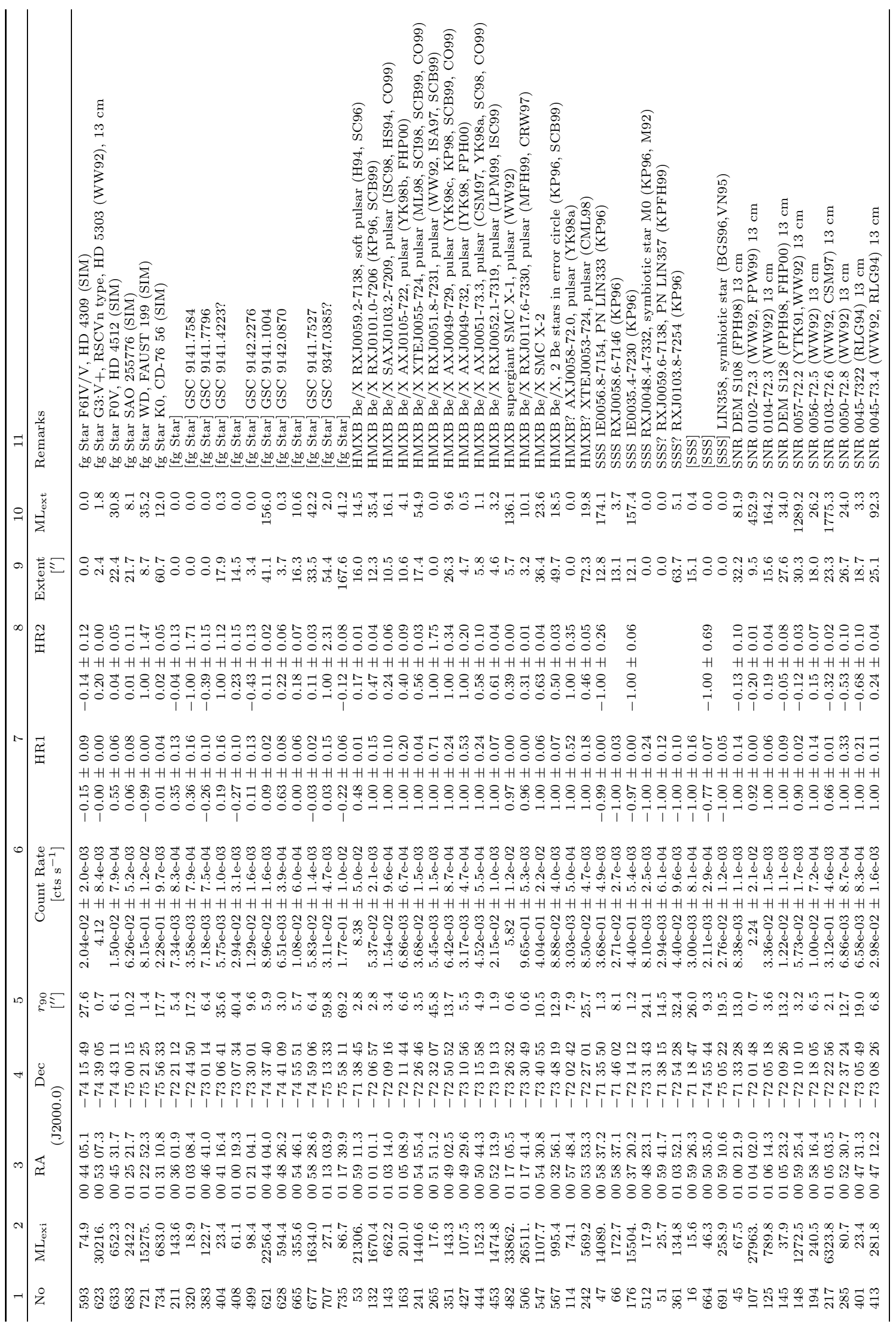


Table 7. continued

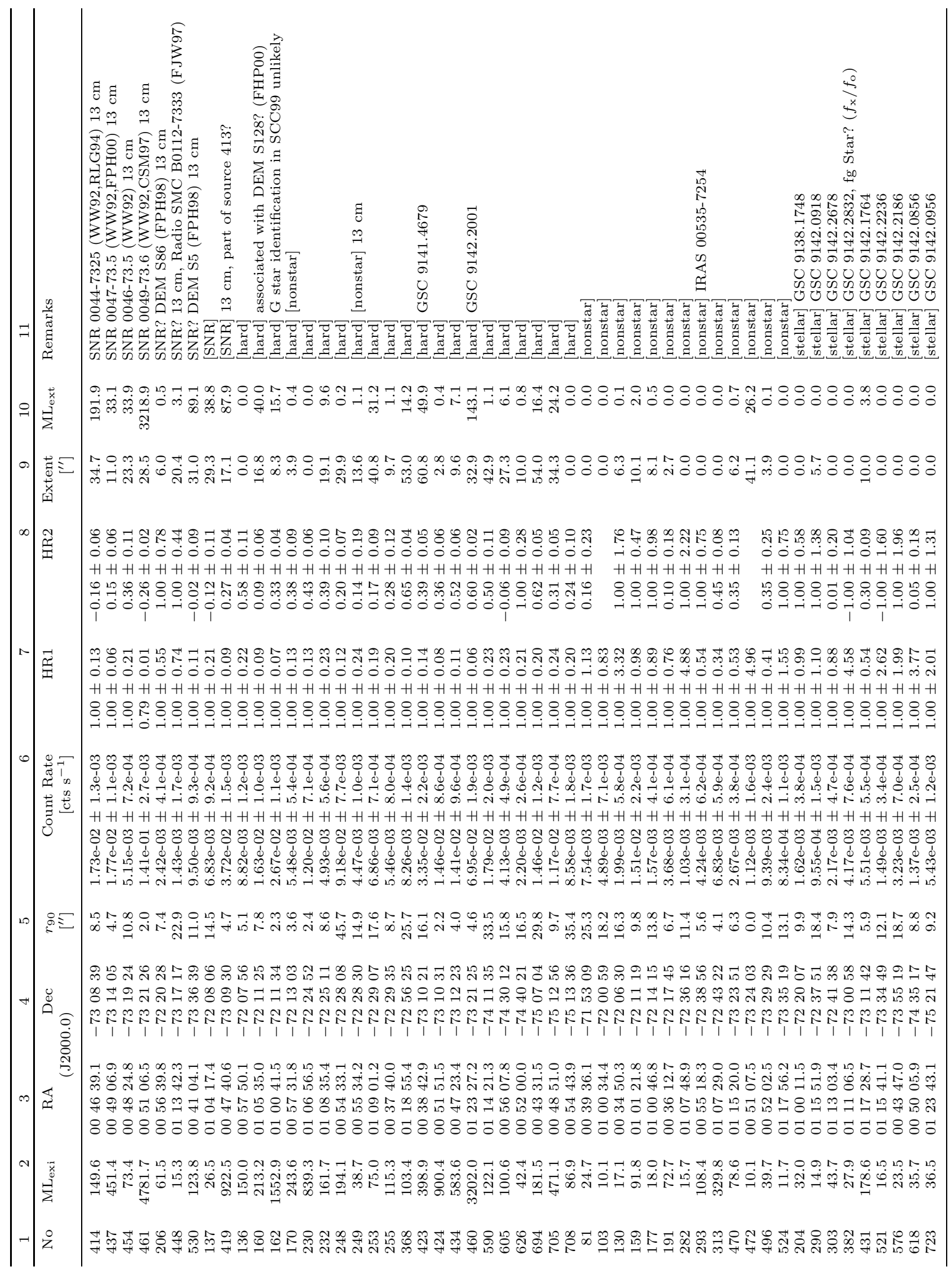

